\title{
Time-Delay Latency of Resting-State Blood Oxygen Level-Dependent Signal Related to the Level of Consciousness in Patients with Severe Consciousness Impairment
}

\author{
Jorge Rudas, ${ }^{1}$ Darwin Martínez, ${ }^{2,3}$ Gabriel Castellanos, ${ }^{4}$ Athena Demertzi, ${ }^{5}$ Charlotte Martial, ${ }^{6}$ \\ Manon Carriére, ${ }^{6}$ Charlène Aubinet, ${ }^{6}$ Andrea Soddu, ${ }^{7}$ Steven Laureys, ${ }^{6}$ and Francisco Gómez ${ }^{8}$
}

\begin{abstract}
Recent evidence on resting-state functional magnetic resonance imaging (rs-fMRI) suggests that healthy human brains have a temporal organization represented in a widely complex time-delay structure. This structure seems to underlie brain communication flow, integration/propagation of brain activity, as well as information processing. Therefore, it is probably linked to the emergence of highly coordinated complex brain phenomena, such as consciousness. Nevertheless, possible changes in this structure during an altered state of consciousness remain poorly investigated. In this work, we hypothesized that due to a disruption in high-order functions and alterations of the brain communication flow, patients with disorders of consciousness (DOC) might exhibit changes in their time-delay structure of spontaneous brain activity. We explored this hypothesis by comparing the time-delay projections from fMRI resting-state data acquired in resting state from 48 patients with DOC and 27 healthy controls (HC) subjects. Results suggest that time-delay structure modifies for patients with DOC conditions when compared with HC. Specifically, the average value and the directionality of latency inside the midcingulate cortex (mCC) shift with the level of consciousness. In particular, positive values of latency inside the mCC relate to preserved states of consciousness, whereas negative values change proportionally with the level of consciousness in patients with DOC. These results suggest that the $\mathrm{mCC}$ may play a critical role as an integrator of brain activity in $\mathrm{HC}$ subjects, but this role vanishes in an altered state of consciousness.
\end{abstract}

Keywords: disorders of consciousness; functional connectivity; latency, midcingulate cortex; resting-state networks; time delay

\section{Introduction}

$\mathbf{F}$ unctional Connectivity (FC) Is a measure of the level of association between the dynamics of pairs of brain regions. FC estimation often assumes instantaneous synchronization among these dynamics (Sinitsyn et al., 2018; Vergara et al., 2018), that is, two regions are functionally connected if there is a zero-lag temporal statistical dependency between them. One of the milestones resulting from FC analyses was the discovery of resting-state networks (RSNs), which are a set of consistent and reproducible spatial brain patterns that share temporal dy- namics and emerge in the spontaneous brain activity of healthy subjects during resting state (Damoiseaux et al., 2006). FC can also be measured by using alternative brain-image modalities, such as electroencephalography or magnetoencephalography (Imperatori et al., 2019; Lottman et al., 2019); these modalities provide high temporal resolutions compared with functional magnetic resonance imaging (fMRI). Nevertheless, they are limited in spatial resolution because of the constraints imposed by the inverse source reconstruction process (Rincon and Shimoda, 2016). Therefore, fMRI becomes a suitable tool with the right balance between spatial and temporal resolution to

\footnotetext{
${ }^{1}$ Institute of Biotechnology, Universidad Nacional de Colombia, Bogotá, Colombia.

${ }^{2}$ Department of Computer Science, Universidad Nacional de Colombia, Bogotá, Colombia.

${ }^{3}$ Department of Computer Science, Universidad Central de Colombia, Bogotá, Colombia.

${ }_{5}^{4}$ Department of Physiological Sciences, Facultad de Medicina, Pontificia Universidad Javeriana, Bogotá, Colombia.

${ }^{5}$ Physiology of Cognition Research Lab, GIGA-Consciousness, GIGA Institute, University of Liege, Liège, Belgium.

${ }^{6}$ Coma Science Group, GIGA Consciousness, University of Liège, Liège, Belgium.

${ }^{7}$ Department of Physics and Astronomy, University of Western Ontario, London, Ontario.

${ }^{8}$ Department of Mathematics, Universidad Nacional de Colombia, Bogotá, Colombia.
} 
compute brain FC (van den Heuvel and Pol, 2010), which has also been previously explored in patients with disorders of consciousness (DOC) (Martinez et al., 2020).

RSNs are the spatial structure of blood oxygen leveldependent (BOLD) signal (Damoiseaux et al., 2006), and they are particularly crucial for studying DOC. DOC encompasses a set of clinical conditions where consciousness has been severely affected by brain damage (Laureys and Schiff, 2012). DOC includes patients with minimally consciousness state (MCS) who show fluctuating evidence of consciousness but remain unable to reproduce this response consistently, and patients with unresponsive wakefulness state (UWS) who have spontaneous eye-opening and independent vital functions. However, they cannot functionally communicate their thoughts or feelings and remain unresponsive to external stimuli (Giacino et al., 2002; Laureys and Schiff, 2012; Laureys et al., 2010). Changes in the intra-inter properties of some of these RSNs relate to alterations in the levels of consciousness (Boerwinkle et al., 2019; Demertzi et al., 2014; Threlkeld et al., 2018). Moreover, DOC conditions link to deficits in global spatial RSNs organization, which seem to result in the inabilities to establish long-distance functional connections (Sitaram et al., 2019). Other evidence from resting-state dynamic FC suggests that changes in the level of consciousness relate to a particular temporal pattern of low-phase coherence (Demertzi et al., 2019). These works suggest a primary role of the brain temporal patterns in the emergence of consciousness. Despite these advances in the comprehension of the consciousness mechanisms, recent developments in the understanding of the temporal structure of spontaneous brain activity suggest that resting-state FC analyses supported by an instantaneous synchronization assumption may only provide a limited view of the brain functioning (Mitra and Raichle, 2018; Mitra et al., 2015b). Therefore, this zero-lag view is probably insufficient to understand a complex phenomenon such as loss of consciousness (Mitra and Raichle, 2018).

Recent evidence shows that intrinsic brain activity also has a temporal structure (Majeed et al., 2011; Mitra et al., 2014). Notably, the FC, taking into account different levels of latency or time delays between the dynamics of pairs of brain regions, results in highly reproducible and consistent patterns of connectivity (Mitra et al., 2014; Meszlenyi et al., 2017; Siegel et al., 2016). In particular, two types of independent delay processes exist in healthy subjects (Mitra et al., 2014): short temporal delays, which are mainly related to the kinetics of neurovascular coupling and are present in scales from microseconds to milliseconds, and long temporal delays, linked to neuronal processes, which emerge in time scales of milliseconds to seconds (Mitra et al., 2014). Brain regions exhibiting these time delays may fall into two different categories: sources or sinks of lagged activity of neuronal origin (Mitra et al., 2014; Mohajerani et al., 2013). When two time series (A and B) are studied by using this framework, A time series is assumed time fixed, and B is shifted $T$ units in time respect to the first one. Then, the $T$ value that maximizes the correlation between both time series corresponds to the lagged value between them. If this $T$ value is lower than zero $(\leq 0)$, that means $\mathrm{B}$ is lagged in time respect to $\mathrm{A}$; thus, B exists at time $t+T$ and is considered a source (lag), and A exists at time $t$ and is a sink (late). Therefore, these sources and sinks may play the role of origins and destinations of the brain communication flow, respectively, that is, if a source brain region is delayed with respect to a sink, then, this region is likely transmitting information to the sink (Mitra and Raichle, 2016; Mitra et al., 2015a). Thus, due to source regions being time-lagged with respect to most other brain regions, these have been related to the propagation of intrinsic brain activity (Deshpande et al., 2011; Lin et al., 2017; Mitra et al., 2014; You et al., 2013). In contrast, sink regions are likely receptors of that brain activity; then, their function is probably related to the information integration, or the induction of changes in the information flow that occur primarily via modulation of the information (Mitra and Raichle, 2018; Mitra et al., 2018).

The characterization of the temporal latency structure in resting may represent a complementary tool for studying brain communication patterns by using fMRI BOLD signals (Mitra and Raichle, 2018). This approach would be highly relevant for the study of altered state of consciousness, for which the transmission, processing of information, as well as the complexity of communication seem disrupted, as suggested by evidence from transcranial magnetic stimulation (Casarotto et al., 2016) and audio stimulus fMRI studies (Naci et al., 2018). By using the time-delay approach, different source or sink brain regions related to high-order functions, such as perception, working memory, and processing of the stimulus, required for the emergence of consciousness, could be further investigated by analyzing their time-delay behaviors (Basar and Düzgün, 2016; Persuh et al., 2018). Similarly, the understanding of temporal latencies in the communication processes involved in stimulus response (Havlicek et al., 2017; Vodrahalli et al., 2018), a function strongly altered in patients with UWS, may also be benefited from this approach.

The lagged temporal structure of the resting-state BOLD signal and its association with different functional systems was previously investigated in healthy controls (HC) (Mitra et al., 2014). Nevertheless, possible changes in these timedelay processes during an altered state of consciousness remain poorly understood. In this study, we explore the role of the lagged processes in the consciousness mechanisms in patients with DOC. We hypothesized that due to disruptions in high-order functions and alterations of the brain communication flows, the time-delay structure of spontaneous brain activity in the resting state of patients with DOC changes. To investigate this hypothesis, we explored the time-delay FC for gray matter regions of interest (ROI) in healthy subjects and DOC patients.

Our results suggest that loss of consciousness in these patients is related to exchanges in the roles of source and sink for particular regions, evidencing a significant reorganization of the brain communication flows during these states. In addition, the average value and directionality of latency inside the midcingulate cortex (mCC) also change with the level of consciousness. In particular, latency values inside mCC positively correlate with the level of consciousness in patients with DOC. Our results point to a new property associated with a breakdown in brain communication in patients with DOC. This study also shows a novel analytic tool for characterizing the level of consciousness in patients with DOC, the temporal structure of the resting-state BOLD signal, and particularly, the time-delay properties of resting-state fMRI (rs-fMRI). Finally, our findings highlight the relevance of considering the temporal structures compared with traditional nonzero lags FC to explore brain dynamics in this kind of patients. 


\section{Materials and Methods}

\section{Participants}

Data from 75 subjects were collected for this study at the University Hospital of Liège, Belgium: $27 \mathrm{HC}$ and 48 patients with DOC (24 patients with MCS and 24 with UWS; 31 men and 18 women; mean age 47; range 14-87; 26 of nontraumatic etiology including anoxic, 11, cerebrovascular accident, 9, hemorrhage, 5, seizure, 1, metabolic; 20 of traumatic etiology and 2 of mixed etiology) (for more details, see Supplementary Data S1). All patients were clinically examined and diagnosed based on repeated Coma Recovery Scale-Revised (CRS-R) assessments, as it was recently recommended by Wannez et al. (2017). Written informed consent to participate in the study was obtained from all subjects and legal surrogates of the patients. The HC were instructed to close their eyes, relax without falling asleep, and refrain from any structured thinking (e.g., counting, singing). The same instructions were given to patients, but due to their cognitive and physical impairments, we could not adequately control for a prolonged eyeclosed yet awake scanning session.

\section{Neuroimaging data acquisition and preprocessing}

For each subject, functional and structural images were acquired by using a 3T scanner manufactured by Siemens Medical Solution. In particular, a structural T1 image for anatomical reference and 300 fMRI volumes multislice T2*-weighted functional images (32 slices; voxel size: $3 \times 3 \times 3 \mathrm{~mm}$; matrix size $64 \times 64 \times 32$; repetition time $=$ $2000 \mathrm{~ms}$; echo time $=30 \mathrm{~ms}$; flip angle $=78^{\circ}$; field of view $=$ $192 \times 192 \mathrm{~mm}$ ) were obtained. Three initial volumes were discarded to avoid T1 saturation effects. rs-fMRI was processed by using SPM8. Preprocessing included: manual realignment, automatic realignment, co-registration of functional onto structural data, segmentation of structural data, normalization into Montreal Neurological Institute (MNI) space, and spatial smoothing with a Gaussian kernel of $8 \mathrm{~mm}$. Framewise displacements analysis was used as suggested (Power et al., 2012) to evaluate the data quality acquisition based on head movements (Supplementary Data S2). In addition, head motion correction included large and rapid motions detection, volume exclusion, and it was applied by using the ArtRepair toolbox for SPM (Demertzi et al., 2014; Mazaika et al., 2009). fMRI volumes with a mean deviation of head movements over a threshold were excluded in the future steps. In this case, the threshold used was $1.5 \%$ with respect to the global mean movements, as suggested by Mazaika et al. (2009). The spurious variance was reduced by regression of nuisance waveforms obtained from time series extracted from regions of noninterest, in particular, white matter and cerebrospinal fluid. Finally, these nuisance regressors included the averaged BOLD time series over the whole brain, as suggested by Fox et al. (2005).

\section{Estimating time-delay projections}

FC estimation commonly involves the computation of Pearson correlation or cross-covariance among BOLD time series (Abrol et al., 2017; Eslami and Saeed, 2018; Madsen et al., 2017). A generalization of cross-covariance function, which considers delays among BOLD time courses to study the latency phenomenon (Mitra et al., 2014), was the base for the proposed analysis. This time-delay formulation of the cross-covariance function corresponds to

$$
C_{t_{i}, t_{j}}(\tau)=\frac{1}{T} \int t_{i}(t+\tau) t_{j} d t
$$

where $t_{i}$ and $t_{j}$ are pairs of filtered time series [bandpass Butterworth filter with cut-off frequencies set at 0.05 and $0.1 \mathrm{~Hz}$ (Demertzi et al., 2014)] for two ROI $i$ and $j, \tau$ is the level of lag between these two-time series (in units of time), and $T$ is the size of the integration interval, in this case, the time series length.

Let $\tau_{t_{i}, t_{j}}$ equal the $\tau$ value that maximizes $C_{t_{i}, t_{j}}(\tau)$ for the proposed analysis $\tau$ range between -3 and $3 \mathrm{sec}$, an interval previously used to characterize neuronal lags (Mitra and Raichle, 2016; Mitra et al., 2014). Then, given $n$ ROIs and their respective time series $\left\{t_{1}, t_{2}, \ldots, t_{n}\right\}$, an $n \times n$ time delay matrix (TDM) was defined:

$$
T D M=\left[\begin{array}{cccc}
\tau_{t_{1}, t_{1}} & \tau_{t_{1}, t_{2}} & \ldots & \tau_{t_{1}, t_{n}} \\
\tau_{t_{2}, t_{1}} & \tau_{t_{2}, t_{2}} & \ldots & \tau_{t_{2}, t_{n}} \\
\vdots & \vdots & \ddots & \vdots \\
\tau_{t_{n}, t_{1}} & \tau_{t_{n}, t_{2}} & \ldots & \tau_{t_{n}, t_{n}}
\end{array}\right] .
$$

This matrix summarizes all the possible time delays between pairs of brain regions. Note that $\tau_{t_{i}, t_{j}}=-\tau_{t_{j}, t_{i}}$, that is, TDM is anti-symmetric, and all elements in the diagonal of TDM are necessarily zero because any time series has zero lag with itself. Nikolić (2007) introduced the TDM to represent the time-delay structure of the spatiotemporal processes. Recently, Mitra et al. (2014) explored this time-delay representation for fMRI data. TDM can be transformed into a onedimensional $(1 \times n)$ vector called time-delay projection $\left(\mathrm{TD}_{\mathrm{p}}\right)$ (Nikolić, 2007), thus:

$$
\mathrm{TD}_{\mathrm{p}}=\frac{1}{n}\left[\sum_{j=1}^{n} \tau_{t_{1}, t_{j}}, \ldots, \sum_{j=1}^{n} \tau_{t_{n}, t_{j}}\right] .
$$

Given that $\mathrm{TD}_{\mathrm{p}}$ is significantly transitive, hence, it contains dominant and consistent lag relationships (Mitra et al., 2014). $\mathrm{TD}_{\mathrm{p}}$ represents the average latency for each ROI compared with the other ROIs in a particular subject. To reduce the computational complexity, we defined ROIs as a set of nonoverlapped cubic portions of the brain with $216 \mathrm{~mm}^{3}$ (27 voxels with voxel size $2 \times 2 \times 2 \mathrm{~mm}$ ) (Mitra et al., 2014). The level of similarity among time series inside each ROI is high for each group (mean Pearson correlation coefficient was close to 0.8) (Supplementary Data S3). Therefore, it is reasonable to assume that this spatial reduction methodology does not affect the estimation of the time-delay approach. TDM was estimated only in the ROIs with at least $50 \%$ of voxel inside the gray matter. Finally, $\mathrm{TD}_{\mathrm{p}}$ maps can be visualized over a 3D image for a graphic representation in the so-called latency map. In these maps, the negative values corresponded to source brain regions and positive values to the sink brain regions. Average $\mathrm{TD}_{\mathrm{p}}$ maps were characterized in $\mathrm{HC}$ by using a similar approach in Mitra et al. (2014), but, as far as we know, this work is reporting for the first time a characterization of resting-state time-delay properties in patients with DOC. The average $\mathrm{TD}_{\mathrm{p}}$ map is a suitable approach to summarize the typical and predominant latency projections in pathological and healthy brain conditions, as suggested by Mitra et al. (2014) and Weng et al. (2018). 


\section{Second-level analysis of the time-delay projections}

A second-level analysis on individual $\mathrm{TD}_{\mathrm{p}}$ was performed to compare HC subjects versus patients with DOC at ROI level. Comparisons were performed with an unpaired $t$-test, and differences were visualized by using a thresholded $z$-map. Given a possible dependence among ROIs, results were adjusted for multiple comparisons by using the false discovery rate and controlled for cluster size $>10$ ROIs.

\section{Estimating differences among time-delay maps}

The regions obtained from the second-level analysis that showed any difference among the three groups were further explored to investigate the power of discrimination of the proposed characterization among different levels of consciousness.

To check independence at the group level in the absence of normal distribution, as was previously suggested in the fMRI BOLD time series by Lewis et al. (2005), Mann-Whitney $U$ tests were used, as suggested by Georgiopoulos et al. (2019) and Zimmermann et al. (2019). This test was applied $(p<0.05 / n)$ among the average $z$-values in each timedelay pattern. These computations were Bonferroni corrected for multiple comparisons $(n=10$ relevant regions to compare).

\section{Estimating integration/modulation effect based on seed region}

The integration/modulation effect of discriminative regions over other brain regions was studied by using a seedbased time-delay approach. In particular, the amount of inte- gration/modulation of the most discriminative region was computed over 48 cortical regions. The Harvard-Oxford Cortical Atlas from the Harvard Center for Morphometric Analysis provided these cortical regions. The labels used to describe the cingulate cortex were taken from Vogt et al. (2006) and Palomero-Gallagher et al. (2009). The amount of integration/modulation corresponded to the average time delay value in the most discriminative region (seed) with respect to ROIs in the other cortical regions. Latency values that provided differences among groups were determined by using a Mann-Whitney $U$ test, Bonferroni corrected $(p<0.05 / n$ where $n=48$ for 48 cortical regions to compare).

\section{Results}

Results consist of three sections: first, a second-level analysis comparing $\mathrm{TD}_{\mathrm{p}}$ across groups, second, changes in time delays associated to the level of consciousness for brain regions with significant differences, and finally, the timedelay integration/modulation influence of the $\mathrm{mCC}$, a region that resulted in a highly related level of consciousness, over other brain cortical regions.

\section{Resting-state time-delay projection maps in patients with $D O C$}

Figure 1 shows the average time-delay maps estimated for HC (top), MCS (center), and UWS (bottom) groups. The duration of these lags ranged in $\pm 0.2 \mathrm{sec}$ for $\mathrm{HC}$ and UWS, $\pm 0.1 \mathrm{sec}$ for MCS (see value's bar in Fig. 1). Average $\mathrm{TD}_{\mathrm{p}}$ for $\mathrm{HC}$ and patients with UWS were remarkably different.
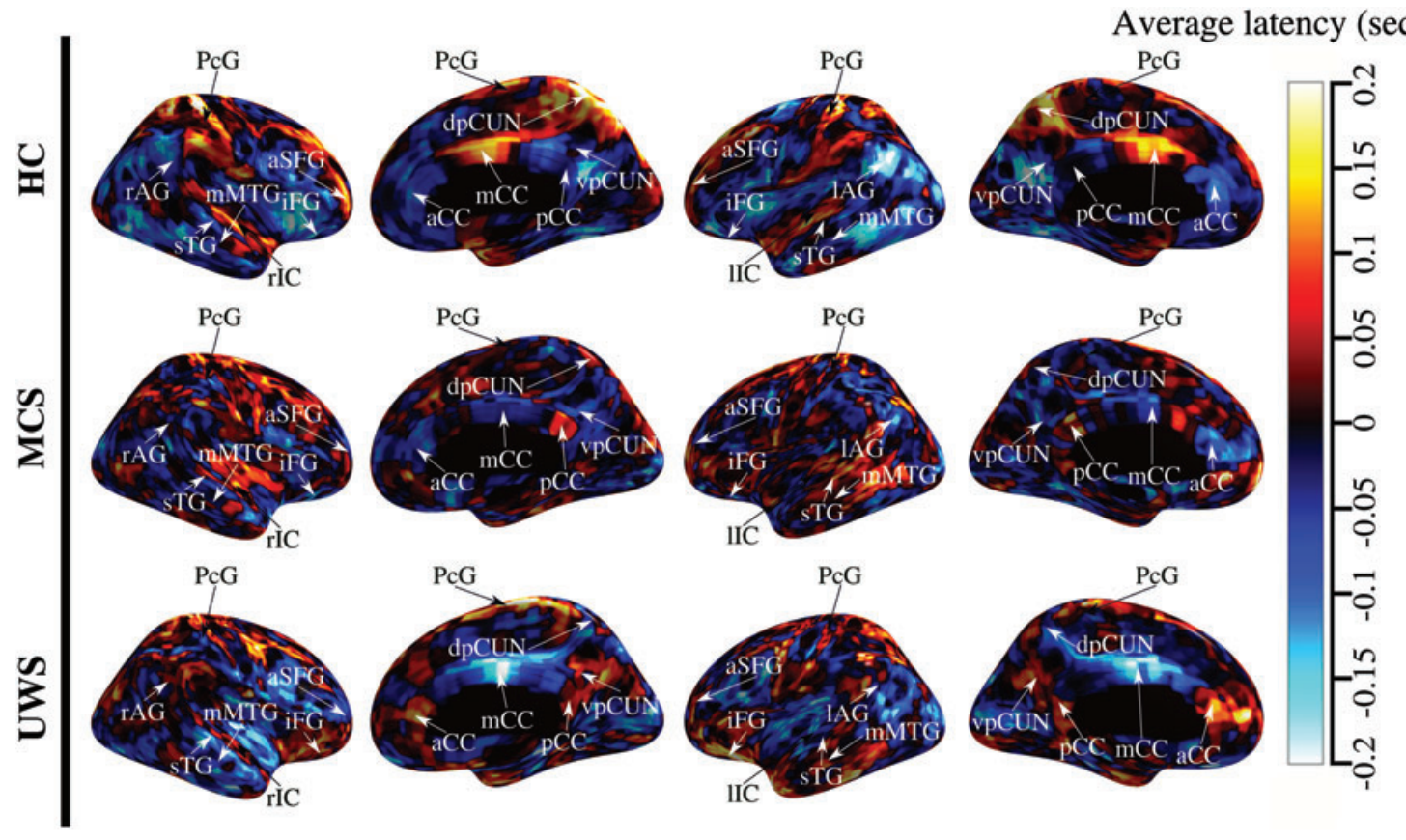

FIG. 1. Average $\mathrm{TD}_{\mathrm{p}}$ maps for different groups. Rows show the average $\mathrm{TD}_{\mathrm{p}}$ for $\mathrm{HC}$ subjects (top), patients with MCS (center) and UWS (bottom), respectively. The color in these plots corresponds to the level of latency in seconds for a particular brain region. Red to yellow areas refer to sink brain regions, and blue to cyan areas refer to source brain regions. The range of latency ranged around $\pm 0.2 \mathrm{sec}$ for HC and UWS, whereas it was around $\pm 0.1 \mathrm{sec}$ for MCS. HC, healthy subjects, MCS, minimally consciousness state; $\mathrm{TD}_{\mathrm{p}}$, time-delay projection; UWS, unresponsive wakefulness state. Color images are available online. 
In particular, a large differentiation between time-delay sources (cyan regions) and sinks (yellow regions) appeared for these two groups, but not in patients with MCS. More specifically, for the $\mathrm{HC}$ group, paracentral gyrus $(\mathrm{PcG})$, precuneus cortex, dorsal division (dpCUN), left-right insular cortex (1rIC) superior temporal gyrus (sTG), and mCC emerged as sinks of the bilaterally temporal latency, and precuneus cortex, ventral division (vpCUN), inferior frontal gyrus (iFG), anterior cingulate cortex $(\mathrm{aCC})$, posterior cingulate cortex (pCC), medial middle temporal gyrus (mMTG), and leftright angular gyrus (1-rAG) resulted as sources of the temporal latency. In patients with UWS, the mCC and 1-rIC exhibited a source behavior, whereas anterior superior frontal gyrus (aSFG) and iFG performed the role of sinks. Finally, patients with MCS did not show any consistent sources or sinks brain regions.

\section{Group-level differences in time-delay maps}

Figure 2 shows the second-level analysis of the $\mathrm{TD}_{\mathrm{p}}$ maps. This analysis aims at identifying possible differences in the latency maps across subjects with different levels of consciousness. As observed, when comparing HC subjects with patients with MCS, mCC and dpCUN changed bilaterally from sink to source. Further, pCC, left angular gyrus (1AG), and posterior superior frontal gyrus (pSFG) interchanged their roles from sources to sinks. A similar interchange of roles emerged when comparing $\mathrm{HC}$ subjects and patients with UWS. Specifically, mCC, dpCUN, and aSFG showed changes from sinks to sources, and pSFG, 1AG, mMTG, $\mathrm{iFG}$, and pCC showed changes from sources to sinks. Moreover, a reduction in the size and number of brain regions with differences inside the time-delay maps resulted when comparing patients with MCS with patients with UWS. Notably, rIC shifted from sink to source, and $\mathrm{iFG}$ and $\mathrm{PcG}$ shifted to sinks.

A complementary analysis to further study the differences between all different states of consciousness was performed. This analysis focused on the 10 brain regions that showed any difference among subject groups (Fig. 2). Figure 3 shows the relationship between latency values in these regions and the level of consciousness. iFG, mMTG, 1AG, dpCUN, and mCC showed significant differences $(p<0.01)$ among $\mathrm{HC}$ and both groups of patients. mCC showed differences across the three comparisons. In particular, positive values of latency inside mCC related to a preserved state of consciousness $(p<0.001)$, and negative values of latency inside

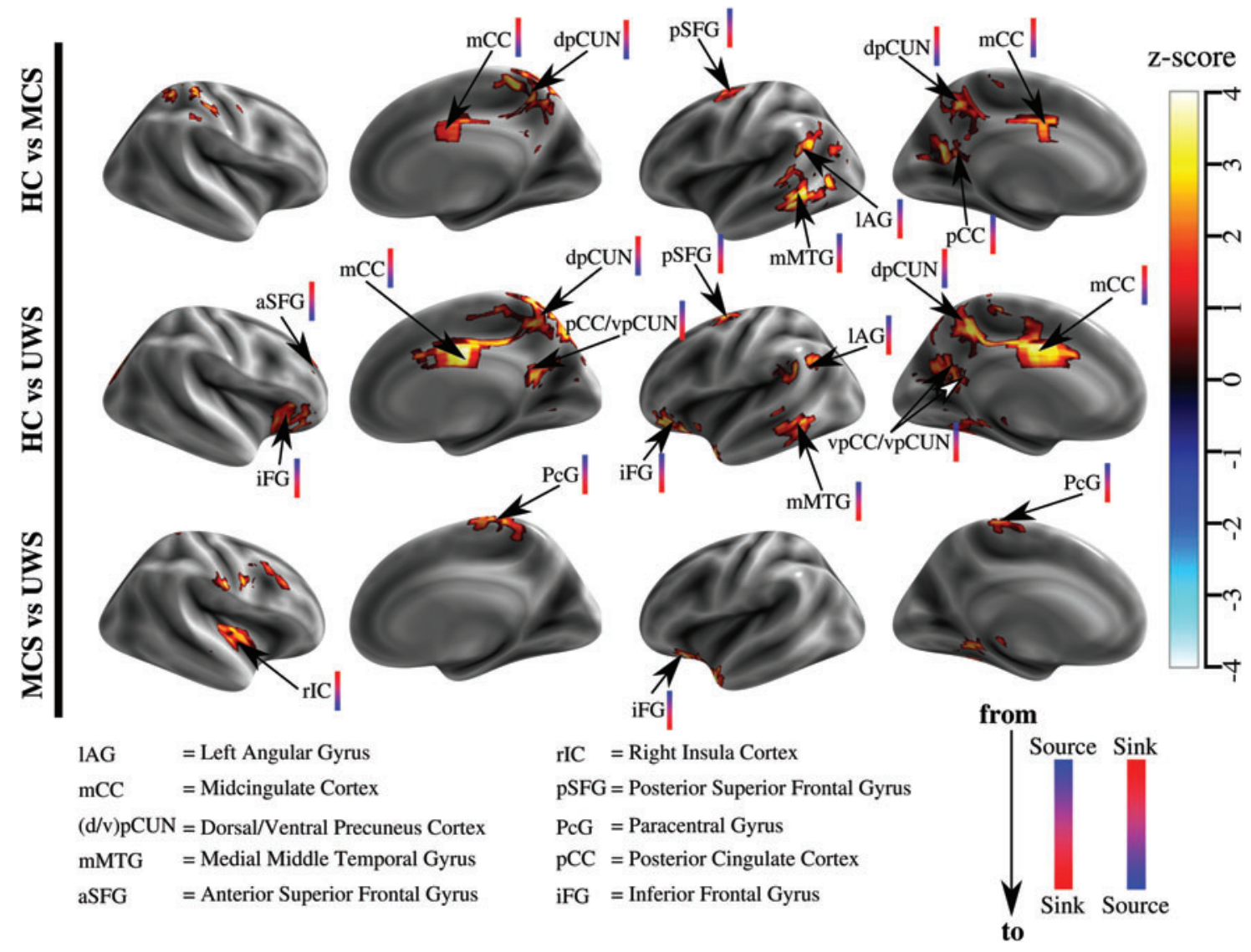

FIG. 2. Differences among $\mathrm{TD}_{\mathrm{p}}$ maps for $\mathrm{HC}$, MCS, and UWS using $z$-maps. Three comparisons are shown: HC versus MCS, HC versus UWS, and MCS versus UWS. The values for each ROI in the thresholded z-map represent the level of differentiation for two groups (FDR $<0.05$ and controlled for cluster size $>10$ ROIs). The color bar close to brain region abbreviation indicates the kind of change of direction. In particular, bars from blue to red indicate changes from source to sink, and bars from red to blue indicate changes from sink to source. FDR, false discovery rate; ROI, regions of interest. Color images are available online. 

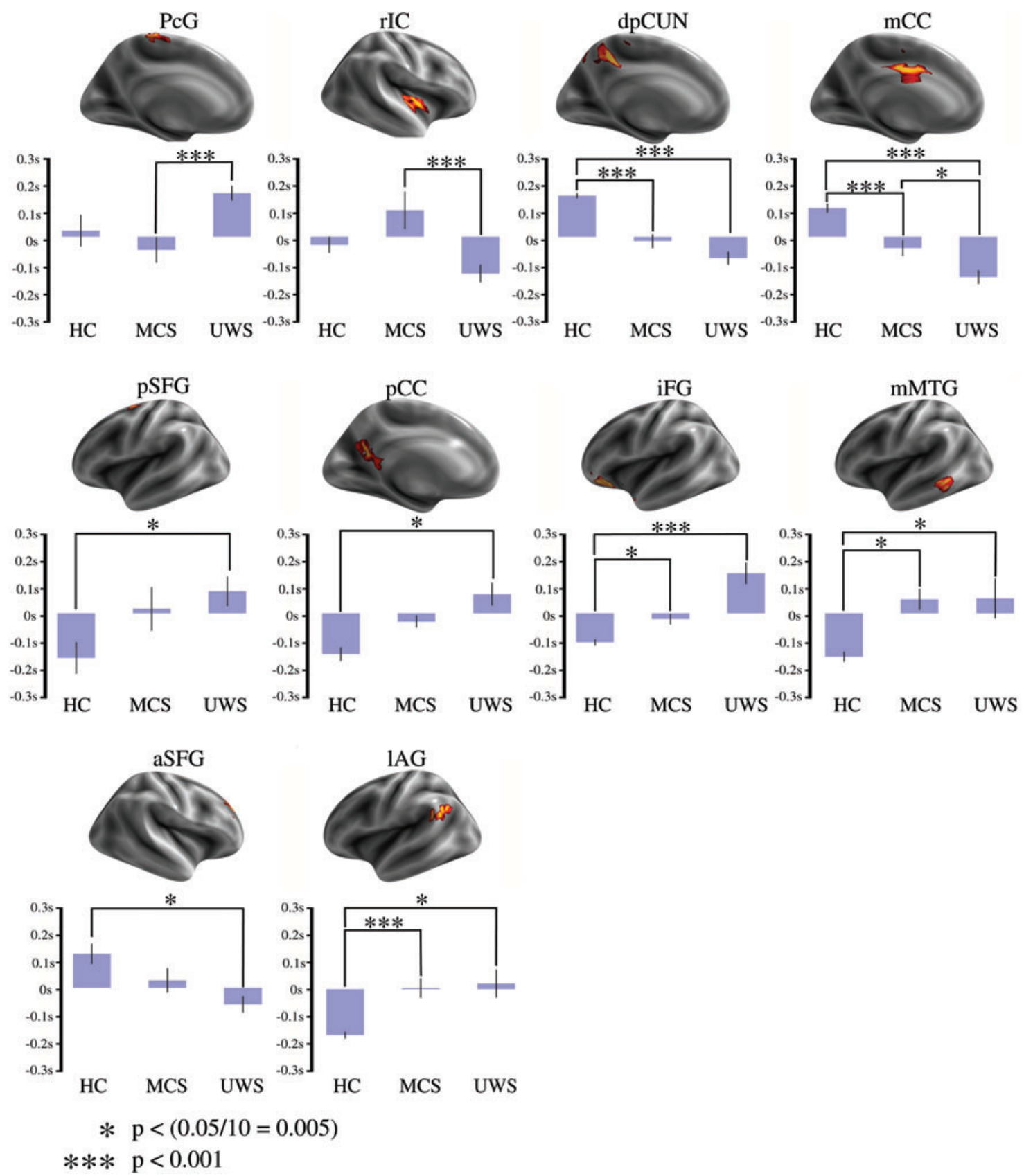

FIG. 3. Average $\mathrm{TD}_{\mathrm{p}}$ values for 10 relevant brain regions and levels of consciousness. Upper plots show the brain regions, which are significantly different among groups. Lower plots show bars with the average values of $\mathrm{TD}_{\mathrm{p}}$ in the 10 brain regions. $\mathrm{TD}_{\mathrm{p}}$ showed significant differences $(p<0.05$ corrected for 10 possible comparisons) for HC subjects compared with DOC patients in the dpCUN, mCC, iFG, mMTG, and 1AG. Remarkably, mCC showed differences among all groups. DOC, disorders of consciousness; dpCUN, precuneus cortex, dorsal division; iFG, inferior frontal gyrus; mCC, midcingulate cortex; mMTG, middle temporal gyrus. Color images are available online.

mCC related to an altered state of consciousness. Besides, these negative time-delay latency values decreased proportionally with the severity of the DOC condition.

\section{Integration of lagged activity to $\mathrm{mCC}$}

The latency values inside $\mathrm{mCC}$ were highly discriminative of the level of consciousness. Particularly, mCC emerged as a sink in $\mathrm{HC}$ subjects (mean: $0.1002 \mathrm{sec}$, variance: $0.0137 \mathrm{sec}$ ) and its influence decreased in patients with
MCS (mean: $-0.037 \mathrm{sec}$, variance $0.0041 \mathrm{sec}$ ), until vanishing in patients with UWS (mean: $-0.138 \mathrm{sec}$, variance: $0.0072 \mathrm{sec}$ ). This trend (Fig. 3, mCC plot) was statistically significant for $\mathrm{HC}$ versus MCS, $\mathrm{HC}$ versus UWS, and MCS versus UWS

To further study these differences, the dependencies between mCC and 48 cortical regions (Englot et al., 2017) were computed by the seed-based time-delay latency approach. Figure 4 shows the regions with significantly different associations of latency using $\mathrm{mCC}$ as seed. Specifically, the $\mathrm{mCC}$ 


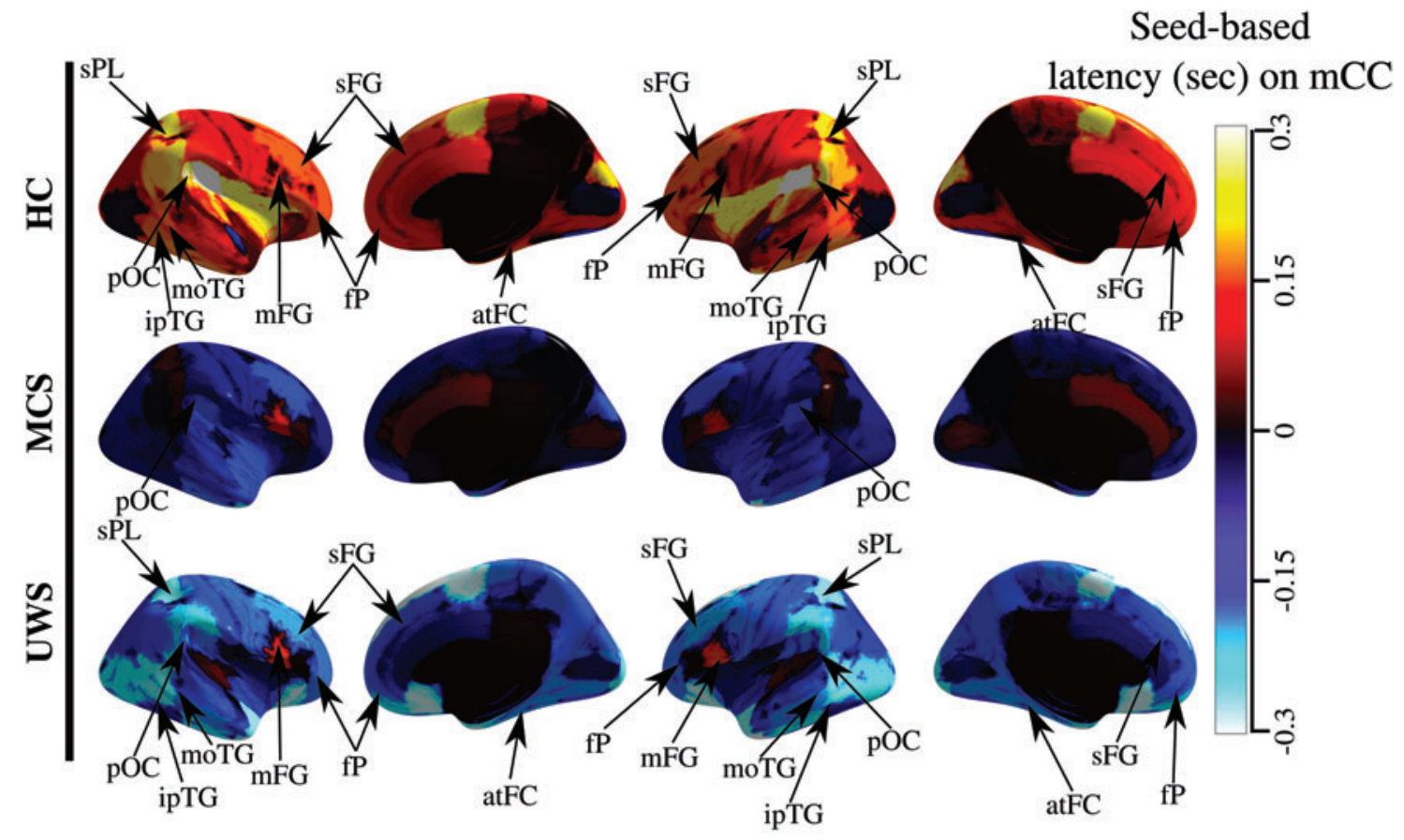

HC vs UWS $*$

Temporal Gyrus, Middle, temporooccipital part (moTG)

Temporal Gyrus, Inferior, posterior division (ipTG)

Middle Frontal Gyrus (mFG)

Frontal Pole (fP)

Superior Parietal Lobule (sPL)

Parietal Operculum Cortex (pOC)

Superior Frontal Gyrus (sFG)

Temporal Fusiform Cortex, Anterior division (atFC)

$* \mathrm{p}<(0.05 / 48=0.001)$

FIG. 4. Integration of the latency activity to $\mathrm{mCC}$. Each row illustrates the average time-delay latency values among mCC (seed) and other cortical regions for HC, MCS, and UWS groups, respectively. The bar's color represents the time-delay latency levels. Blue to cyan colors correspond to sources brain regions and red to yellow correspond to sink brain regions. In $\mathrm{HC}$ subjects, mCC seems to be a sink of the time-delay latency activity for almost all cortical regions. This contrasts with MSC and UWS, where mCC lost this behavior. Eight relationships show statistical differences between HC and UWS, and one relationship shows a difference between HC subjects and patients with MCS $(p<0.05$ corrected for 48 possible comparisons). Color images are available online.

appeared as a sink of latency, for almost all brain cortical areas explored in the $\mathrm{HC}$ subjects. This property vanishes in an altered state of consciousness (see the predominant positive values in $\mathrm{HC}$ subjects in Fig. 4). Besides, the associations among $\mathrm{mCC}$ and frontal pole (fP), superior and middle frontal gyrus, middle and inferior temporal gyrus, superior parietal lobule (sPL), anterior temporal fusiform cortex (atFC), and parietal operculum cortex (pOC) were different between HC subjects and patients with UWS $(p<0.001)$. Finally, the level of latency between $\mathrm{mCC}$ and pOC was different in $\mathrm{HC}$ subjects compared with patients with MCS $(p<0.001)$.

\section{Discussion}

\section{Summary of findings}

In this study, we used time-delay projections to characterize the temporal structure of the resting-state signal in patients with DOC. Nikolić et al. (2007) introduced these time-delay projections for studying the temporal lag structure of the BOLD signal. Posteriorly, Mitra et al. (2014) evidenced the existence of a reproducible lag structure in the resting state of $\mathrm{HC}$ subjects. In this work, we studied possible changes in this lag structure linked to the severity of loss of consciousness in patients with DOC. Results showed differentiation between sources and sinks of the time-delay structure in $\mathrm{HC}$ subjects and patients with UWS, but not in patients with MCS, as observed in Figure 1. Interestingly, different brain regions lost their role as a source or sink of the latency structure in patients with DOC (Fig. 2). Besides, various brain regions showed a linear trend of the latency values associated with the level of consciousness. Notably, mCC's latency values provided differentiation among the three groups (Fig. 3). mCC also showed an integrator/modulator behavior, that is, its primary role was forwarded in time respect to multiple cortical brain regions in $\mathrm{HC}$ subjects, in contrast to patients with DOC, where this integration/modulation effect vanished (Fig. 4). Finally, a set of latency values among 
$\mathrm{mCC}$ and other cortical brain regions showed differences between HC subjects and patients with UWS.

\section{Neurophysiology of the time-delay maps in patients with DOC}

In the past two decades, changes in the fMRI BOLD signal have been linked to blood flow variations (Buxton, 2013). In particular, the changes in the local concentration of deoxyhemoglobin, and consequently, the paramagnetic effect produced by them result in BOLD signal variations (Buxton, 2013). At micro-scale, these changes relate to modifications in local field potentials at cellular level (Logothetis et al., 2001), cellular metabolism (Magistretti and Allaman, 2015), and aerobic glycolysis (Vaishnavi et al., 2010), among other mechanisms. These changes are also linked to variations of the underlying neural activity or modifications of the vascular hemodynamic response (Bandettini et al., 1992; Logothetis et al., 2001). The mechanisms underlying the changes of the slow propagation of intrinsic cortical activity are still not well understood. Positron emission tomography studies have shown that metabolic activity in some cortical areas as the cingulate cortex reverses their magnitude during the wake-sleep cycle. Especially, metabolic activation during rapid eye movement (REM) sleep exceeds other states levels in the aCC, and it is reduced in the pCC (Braun et al., 1997; Maquet et al., 1996). In addition, several studies in patients with UWS show a widespread decrease in brain metabolism mainly encompassing $\mathrm{aCC}, \mathrm{pCC} / \mathrm{pCUN}$, and lateral associative cortices (BeuthienBaumann et al., 2003; Laureys et al., 1999; Nakayama et al., 2006).

Recently, Mitra et al. (2014, 2015a) reported the existence of a lag structure in the resting-state BOLD signal with a possible neural basis. These findings are critical for the interpretation of our results, because they suggest a possible relationship between functional brain communication and the lag structure in resting state (Mitra et al., 2014, 2015a, 2018). Mitra et al. (2018) found that the direction of cortical activity in mice is concordant across calcium/hemoglobin imaging and electrophysiology, and they also showed that the reversal of the predominant direction of travel of cortical activity is state dependent and accomplished by changing the relative weighting and relationships of signals in distinct cortical layers. In addition, the microvascular damage at the cell level, and the reconfiguration of blood flow after a brain injury may also be determinant factors of the latency changes observed in the functional communication in patients with brain damage (Siegel et al., 2016). Therefore, our results can be interpreted from these two perspectives: neuronal and vascular.

From a neurophysiological perspective, we hypothesized that changes in the spatial organization of the time-delay projections maps, observed in Figure 1, may be linked with a functional reconfiguration of resting activity in an altered state of consciousness. Previous works showed that many functional properties change in this group of patients (Demertzi et al., 2014; Demertzi et al., 2015; Marino et al., 2016; Rudas et al., 2014), in particular, FC inside some RSN (Demertzi et al., 2015; Marino et al., 2016), FC among RSN (Rudas et al., 2014), and other RSN intrinsic properties (Demertzi et al., 2014). These spatial changes in rs-fMRI seem to be dependent on the temporal structure. For instance, recently, it was evidenced that the spatial orga- nization of the resting-state BOLD signal, in particular, the RSNs organization, is a consequence of a temporal structure in this signal (Mitra and Raichle, 2016). This fact suggests that latency structure may serve as a complementary way to explore functional changes in this group of patients.

From a vascular perspective, patients after stroke showed a bilateral disruption between hemispheres in the time-delay structure (siegel et al., 2016). This finding suggests that the latency structure may be modulated focally by changes in arterial transit time due to a rerouting of blood flow (Siegel et al., 2016). The population herein studied was affected by multiple etiologies, including anoxic events, cerebrovascular accident, hemorrhage, seizure, and traumatic brain injury (Supplementary Data $\mathrm{S} 1)$. In principle, these etiologies may also produce shifts in the neurovascular coupling. Nevertheless, our results show that the range of latency $( \pm 0.2 \mathrm{sec})$ is similar, at the temporal scale, to the one reported for HC subjects (Mitra et al., 2014). It is important to note that this temporal range is smaller than the latency range reported in patients after stroke ( $>1 \mathrm{sec}$ ) (Siegel et al., 2016). These differences can be related to the diversity in the etiologies in our study. These ranges also suggest that in this context, a neuronal explanation is more suitable to the observed lags, due to the short scale in the range of latency values observed (Fig. 1). Despite that, several questions remain regarding how neuronal and hemodynamic phenomenon in the latency structure relates to consciousness, in particular: Which is the most critical cause of the latency structure of a patient with DOC?

\section{The role of latency activity in patients with DOC}

The implicit assumption in the traditional FC approach is that the correlation between brain regions occurs with zero latency (Schiefer et al., 2018). This assumption has two limitations: First, there is no consideration about the time required for information processing in each brain region, and second, this approach does not provide insights about how brain regions are communicating within themselves or with other brain areas (Mitra and Raichle, 2018). The method herein used aims at overcoming these limitations (Mitra and Raichle, 2018). In particular, given a pair of brain regions, the first region will be delayed with respect to the second one if there is a temporal lag between them. Then, the first region will be a source, and the second one a sink in a communication process (Mitra and Raichle, 2018). Under this interpretation, the time-delay changes (source to sink and sink to source) may relate to variations in the directionality of the FC (Mitra and Raichle, 2016). This directionality has been previously quantified by using causal approximations, such as Granger causality (Duggento et al., 2018) and dynamic causal modeling (Tak et al., 2018). However, these approaches may be inappropriate in large spatiotemporal scales, as in the temporal propagation in the whole brain (Mitra and Raichle, 2016). Therefore, the time-delay structure will constitute a complementary approach to explore the brain information flow and the modulation of higher frequency activity (Mitra et al., 2018).

Our results suggest that $\mathrm{mCC}$ and dpCUN changed from sink to source and pSFG, mMTG, pCC, vpCUN, and 1AG changed from source to sink when comparing $\mathrm{HC}$ subjects versus patients with DOC. These modifications may be associated with a variation of directionality in the FC, changes of brain metabolic activity, or state-dependent changes in the 
temporal structure of intrinsic infra-slow neurophysiological cortical processes during unconsciousness (Mitra and Raichle, 2018; Mitra et al., 2018). Importantly, $\mathrm{mCC}$ is a multisensory premotor region that integrates information from other associative cortical areas (Vogt, 2016). In Vogt's model, the human cingulate cortex is subdivided into at least three sub-regions, the main being the aCC, $\mathrm{mCC}$, and pCC (Palomero-Gallagher et al., 2009), which have been validated in functional and structural connectivity studies (Jin et al., 2018; Yu et al., 2011). Salience network (SN) and the default mode network (DMN) do not usually involve the $\mathrm{mCC}$, but this region is flanked on both sides by aCC and pCC activity. The mCC region differentially receives afferent connections from the parietal cortex that integrate with frontal, insular, temporal, and cingulate information to regulate cognitive and sensorimotor functions (Bush et al., 2000; Vogt, 2016). This region includes the most abundant cingulate neurons [gigantopyramidal neurons of Braak (1976)] that project to the facial nucleus and spinal cord. Gigantopyramidal neurons seem to modulate the motor response of cognitively demanding tasks that involve the face or the upper limbs such as precision grip tasks (Bush et al., 2000; Ehrsson et al., 2000), locomotor-like stepping movements (Martinez et al., 2016), and the orientation of the body in space to vestibular and other sensory stimuli (Caruana et al., 2018). Related to these sensorimotor functions of the cingulate cortex, the activity generated by nociceptive stimuli is located mainly in $\mathrm{mCC}$ and is altered in DOC patients (Laureys et al., 2002). In particular, the linkage between pain processing and movement is severely affected in patients with UWS (Pistoia et al., 2016).

Resting-state and stimulus-based fMRI studies of patients with DOC suggest marked anomalies in cortical connectivity affecting multiple extrinsic and intrinsic networks such as the SN and the DMN (Demertzi et al., 2015). aCC is a structurally functionally heterogeneous sub-region of the $\mathrm{SN}$ associated with many important cognitive and affective functions (Seeley et al., 2007). The SN integrates external sensory stimuli with internal states, and it also has a role in coordinating network dynamics and possibly in switching between the central executive network and the DMN in response to salient stimuli (Goulden et al., 2014). On the other hand, pCC is a primary brain region of the DMN. DMN is related to multiple highlevel brain functions such as self-related mental processes, emotional state, consciousness, and others (Davey et al., 2016). Moreover, DMN is a hub of neural information transmission as the major locus of intrinsically propagated brain activity (Deshpande et al., 2011; Lin et al., 2017; You et al., 2013). Commonly, pCC reduces its internal FC during a complex task and exhibits an increase of functional coupling with task-related regions, in particular, executive control regions such as the dorsolateral prefrontal cortex (Krieger-Redwood et al., 2016). Different studies suggest this particular pattern of increase of FC is linked to pCC, in particular, for semantic tasks (Krieger-Redwood et al., 2016), tasks of working memory (Konishi et al., 2015), creativity (Beaty et al., 2016), and future planning (Gerlach et al., 2011). This increase of communication from/to pCC is also linked to a contribution in paramount aspects of memory retrieval and cognition (KriegerRedwood et al., 2016). Besides, FC among pCC and other brain regions may support the information processing even if its internal activation level is decreasing during a task activity (Krieger-Redwood et al., 2016). Therefore, this evidence suggests that $\mathrm{pCC}$ has a primary role during the communication and processing of information in $\mathrm{HC}$ subjects. We can also interpret the same findings (Figs. 3 and 4) under a causal perspective, as suggested by Mitra and Raichle (2016, 2018). Hence, sink regions, which are forwarded in the time respect to other brain regions, are possibly the destination of brain activity. This approach is also in agreement with the evidence reported by Crone et al. (2015). In this work, key brain regions of the DMN were considered as nodes in a spectral dynamic causal modeling analysis to evaluate the nature of communication flow involved for these regions in patients with DOC. Their results showed that $\mathrm{pCC}$ is the central hub in a causal process inside the DMN in $\mathrm{HC}$ subjects, but this pattern is disrupted in patients with DOC. Therefore, aCC and pCC constitute a primary locus of time-delay sources during resting state in $\mathrm{HC}$ subjects, and they possibly serve as modulator/propagator regions of brain activity. $\mathrm{mCC}$ seems to integrate information from these two sources of brain regions, possibly serving as a sink region of the whole-brain activity in $\mathrm{HC}$ subjects as also evidenced by Mitra et al. (2014), and this role is lost in an altered state of consciousness, as seen in Figure 4.

\section{Methodological considerations}

In this work, we identified three methodological limitations. First, we found different etiologies from patients with brain injury (traumatic event, anoxic event, cerebrovascular accident, hemorrhage, and others) in the population under study; therefore, some patients can present non-neuronal time delay during the functional communication due to the rerouting of blood flow, as suggested by Siegel et al. (2016). This is particularly relevant in etiologies such as cerebrovascular accidents and hemorrhage. Second, fMRI data used in this work have a temporal resolution of $2000 \mathrm{~ms}$, which implies a limitation to capture smaller value in temporal scale for the time-delay approach. To overcome that fact, we used the strategies proposed by Mitra et al. (2014). Then, this strategy based on interpolation of the time series to increase the temporal resolution can introduce a methodological limitation to this study. Third, during the estimation of time-delay maps, we changed the spatial resolution of our data to ROI with $6 \mathrm{~mm}^{3}$ to reduce the computational burden losing spatial resolution. Even though this approach was previously used in a similar context by Mitra et al. (2014), this step can influence our results. Therefore, future works may consider smaller ROI regions to increase the spatial resolution of our approach, fMRI with a high temporal resolution (repetition time $<1 \mathrm{sec}$ ), a homogeneous set of etiologies to reduce the bias for several brain injury conditions, and a seed-based lag maps analysis over the whole brain to explore time-delay relationships among brain entities in DOC conditions.

\section{Conclusions}

This study showed that the time-delay patterns of brain activity are different in patients with DOC with respect to the observed time-delay patterns in $\mathrm{HC}$ subjects. Notably, pSFG, mMTG, pCC, and 1AG changed from sources to sinks, and $\mathrm{mCC}$ and dpCUN changed from sinks to sources of the time-delay structure in DOC patients. Moreover, $\mathrm{mCC}$ seems to be a multi-modal brain region that integrates information from various sources in HC subjects, but this 
role seems to vanish in an altered state of consciousness. We also observed that time-delay value inside mCC seems to be linked to DOC severity. Therefore, these findings indicate a new property based on the level of latency in the brain FC to evaluate the level of consciousness in patients with DOC. Further studies are still required to confirm these findings and explore their potential value for clinical application.

\section{Data and Methods Availability}

The clinical and neuroimaging datasets that emerged from this study are available from the corresponding author on reasonable request. High-quality figures and computational implementation are publicly accessed from the official page of "Computational Modeling of Biological System Group (COMBIOS).",

\section{Authors' Contributions}

J.R., D.M., and F.G. conceived the experiments. J.R. conducted the data analysis. A.D., C.M., M.C., C.A., and S.L. conducted the data acquisition and clinical assessments. J.R. and F.G wrote the article; A.S. and G.C. provided their expertise. All authors provided feedback on the article.

\section{Author Disclosure Statement}

No competing financial interests exist.

\section{Funding Information}

This work was supported by the James McDonnell Foundation, the BIAL Foundation, the Belgian National Funds for Scientific Research (FRS-FNRS), the European Union's Horizon 2020 Framework Programme for Research and Innovation under the Specific Grant Agreement No. 785907 (Human Brain Project SGA2), the Luminous project (EU-H2020fetopenga686764), the European Space Agency, Mind Science Foundation, the French Speaking Community Concerted Research Action, the Belgian inter-university attraction pole, the Public Utility Foundation "Université Européenne du Travail," "Fondazione Europea di Ricerca Biomedica," and the University and University Hospital of Liége. In addition, this work was further supported by the agreement between the Gobernación del Magdalena and COLCIENCIAS (Instituto Colombiano para el Desarrollo de la Ciencia y la Tecnología) for the education of high-quality human capital in the department of Magdalena, the project titled "A functional activity maps for 1.5T fMRI data in resting-state" at Universidad Nacional de Colombia and the project "Caracterización de la conectividad estructural y funcional del sistema reticular ascendente por medio de resonancia magnética con tractografía y BOLD, para la predicción del estado de conciencia en pacientes posreanimación o con lesión cerebral traumática" (Code: 55796) from programa nacional de ciencia, tecnología e innovación en salud of COLCIENCIAS 744.

\section{Supplementary Material}

Supplementary Data S1

Supplementary Data S2

Supplementary Data S3

\section{References}

Abrol A, Damaraju E, Miller RL, Stephen JM, Claus ED, Mayer AR, Calhoun VD. 2017. Replicability of time-varying connectivity patterns in large resting state fMRI samples. Neuroimage 163:160-176.

Bandettini PA, Wong EC, Hinks RS, Tikofsky RS, Hyde JS. 1992. Time course EPI of human brain function during task activation. Magn Reson Med 25:390-397.

Basar E, Düzgün A. 2016. Links of consciousness, perception, and memory by means of delta oscillations of brain. Front Psychol 7:275.

Beaty RE, Benedek M, Silvia PJ, Schacter DL. 2016. Creative cognition and brain network dynamics. Trends Cogn Sci 20:87-95.

Beuthien-Baumann B, Handrick W, Schmidt T, Burchert W, Oehme L, Kropp J, et al. 2003. Persistent vegetative state: evaluation of brain metabolism and brain perfusion with PET and SPECT. Nucl Med Commun 24:643-649.

Boerwinkle VL, Torrisi SJ, Foldes ST, Marku I, Ranjan M, Wilfong AA, Adelson PD. 2019. Resting-state fMRI in disorders of consciousness to facilitate early therapeutic intervention. Neurol Clin Pract 9:e33-e35.

Braak H. 1976. A primitive gigantopyramidal field buried in the depth of the cingulate sulcus of the human brain. Brain Res 109:219-223.

Braun AR, Balkin TJ, Wesenten NJ, Carson RE, Varga M, Baldwin P, et al. 1997. Regional cerebral blood flow throughout the sleep-wake cycle. An H2(15)O PET study. Brain 120 (Pt 7):1173-1197.

Bush G, Luu P, Posner MI. 2000. Cognitive and emotional influences in anterior cingulate cortex. Trends Cogn Sci 4:215222.

Buxton RB. 2013. The physics of functional magnetic resonance imaging (fMRI). Rep Prog Phys 76:096601.

Caruana F, Gerbella M, Avanzini P, Gozzo F, Pelliccia V, Mai $\mathrm{R}$, et al. 2018. Motor and emotional behaviours elicited by electrical stimulation of the human cingulate cortex. Brain 141:3035-3051.

Casarotto S, Comanducci A, Rosanova M, Sarasso S, Fecchio M, Napolitani M, et al. 2016. Stratification of unresponsive patients by an independently validated index of brain complexity. Ann Neurol 80:718-729.

Crone JS, Schurz M, Holler Y, Bergmann J, Monti M, Schmid E, et al. 2015. Impaired consciousness is linked to changes in effective connectivity of the posterior cingulate cortex within the default mode network. Neuroimage 110:101-109.

Damoiseaux JS, Rombouts SARB, Barkhof F, Scheltens P, Stam CJ, Smith SM, Beckmann CF. 2006. Consistent resting-state networks across healthy subjects. Proc Natl Acad Sci U S A 103:13848-13853.

Davey CG, Pujol J, Harrison BJ. 2016. Mapping the self in the brain's default mode network. Neuroimage 132:390-397.

Demertzi A, Antonopoulos G, Heine L, Voss H, Crone J, De Los Angeles C, et al. 2015. Intrinsic functional connectivity differentiates minimally conscious from unresponsive patients. Brain 138:2619-2631.

Demertzi A, Gómez F, Crone J, Vanhaudenhuyse A, Tshibanda L, Noirhomme Q, et al. 2014. Multiple fMRI system-level baseline connectivity is disrupted in patients with consciousness alterations. Cortex 52:35-46.

Demertzi A, Tagliazucchi E, Dehaene S, Deco G, Barttfeld P, Raimondo F, et al. 2019. Human consciousness is supported by dynamic complex patterns of brain signal coordination. Sci Adv 5:eaat7603. 
Deshpande G, Santhanam P, Hu X. 2011. Instantaneous and causal connectivity in resting state brain networks derived from functional MRI data. Neuroimage 54:1043-1052.

Duggento A, Passamonti L, Valenza G, Barbieri R, Guerrisi M, Toschi N. 2018. Multivariate granger causality unveils directed parietal to prefrontal cortex connectivity during taskfree MRI. Sci Rep 8:5571.

Ehrsson HH, Fagergren A, Jonsson T, Westling G, Johansson RS, Forssberg H. 2000. Cortical activity in precision- versus power-grip tasks: an fMRI study. J Neurophysiol 83:528536.

Englot DJ, D'Haese PF, Konrad PE, Jacobs ML, Gore JC, AbouKhalil BW, Morgan VL. 2017. Functional connectivity disturbances of the ascending reticular activating system in temporal lobe epilepsy. J Neurol Neurosurg Psychiatry 88:925-932.

Eslami T, Saeed F. 2018. Fast-GPU-PCC: a GPU-based technique to compute pairwise Pearson's correlation coefficients for time series data-fMRI study. High Throughput 7. DOI: 10.3390/ht7020011

Fox MD, Snyder AZ, Vincent JL, Corbetta M, Van Essen DC, Raichle ME. 2005. The human brain is intrinsically organized into dynamic, anticorrelated functional networks. Proc Natl Acad Sci U S A 102:9673-9678.

Georgiopoulos C, Witt ST, Haller S, Dizdar N, Zachrisson H, Engström M, Larsson EM. 2019. A study of neural activity and functional connectivity within the olfactory brain network in Parkinson's disease. Neuroimage Clin 23:101946.

Gerlach KD, Spreng RN, Gilmore AW, Schacter DL. 2011. Solving future problems: default network and executive activity associated with goal-directed mental simulations. Neuroimage 55:1816-1824.

Giacino JT, Ashwal S, Childs N, Cranford R, Jennett B, Katz DI, et al. 2002. The minimally conscious state: definition and diagnostic criteria. Neurology 58:349-353.

Goulden N, Khusnulina A, Davis NJ, Bracewell RM, Bokde AL, McNulty JP, Mullins PG. 2014. The salience network is responsible for switching between the default mode network and the central executive network: replication from DCM. Neuroimage 99:180-190.

Havlicek M, Ivanov D, Roebroeck A, Uluda? K. 2017. Determining excitatory and inhibitory neuronal activity from multimodal fMRI data using a generative hemodynamic model. Front Neurosci 11:616.

Imperatori LS, Betta M, Cecchetti L, Canales-Johnson A, Ricciardi E, Siclari F, et al. 2019. EEG functional connectivity metrics wPLI and wSMI account for distinct types of brain functional interactions. Sci Rep 9:8894.

Jin F, Zheng P, Liu H, Guo H, Sun Z. 2018. Functional and anatomical connectivity-based parcellation of human cingulate cortex. Brain Behav 8:e01070.

Konishi M, McLaren DG, Engen H, Smallwood J. 2015. Shaped by the past: the default mode network supports cognition that is independent of immediate perceptual input. PLoS One 10: e0132209.

Krieger-Redwood K, Jefferies E, Karapanagiotidis T, Seymour R, Nunes A, Ang JWA, et al. 2016. Down but not out in posterior cingulate cortex: deactivation yet functional coupling with prefrontal cortex during demanding semantic cognition. Neuroimage 141:366-377.

Laureys S, Celesia GG, Cohadon F, Lavrijsen J, León-Carrión J, Sannita WG, et al. 2010. Unresponsive wakefulness syndrome: a new name for the vegetative state or apallic syndrome. BMC Med 8:1-4.
Laureys S, Faymonville ME, Peigneux P, Damas P, Lambermont B, Del Fiore G, et al. 2002. Cortical processing of noxious somatosensory stimuli in the persistent vegetative state. Neuroimage 17:732-741.

Laureys S, Lemaire C, Maquet P, Phillips C, Franck G. 1999. Cerebral metabolism during vegetative state and after recovery to consciousness. J Neurol Neurosurg Psychiatry 67:121.

Laureys S, Schiff N. 2012. Coma and consciousness: paradigms (re)framed by neuroimaging. Neuroimage 61:478-491.

Lewis SM, Jerde TA, Tzagarakis C, Gourtzelidis P, Georgopoulos MA, Tsekos N, et al. 2005. Logarithmic transformation for high-field BOLD fMRI data. Exp Brain Res 165:447-453.

Lin P, Yang Y, Gao J, De Pisapia N, Ge S, Wang X, et al. 2017. Dynamic default mode network across different brain states. Sci Rep 7:46088.

Logothetis NK, Pauls J, Augath M, Trinath T, Oeltermann A. 2001. Neurophysiological investigation of the basis of the fMRI signal. Nature 412:150-157.

Lottman KK, Gawne TJ, Kraguljac NV, Killen JF, Reid MA, Lahti AC. 2019. Examining resting-state functional connectivity in first-episode schizophrenia with 7T fMRI and MEG. Neuroimage Clin 24:101959.

Madsen KH, Churchill NW, Mørup M. 2017. Quantifying functional connectivity in multi-subject fMRI data using component models. Hum Brain Mapp 38:882-899.

Magistretti P, Allaman I. 2015. A cellular perspective on brain energy metabolism and functional imaging. Neuron 86: 883-901.

Majeed W, Magnuson M, Hasenkamp W, Schwarb H, Schumacher EH, Barsalou L, Keilholz SD. 2011. Spatiotemporal dynamics of low frequency BOLD fluctuations in rats and humans. Neuroimage 54:1140-1150.

Maquet P, Peters J, Aerts J, Delfiore G, Degueldre C, Luxen A, Franck G. 1996. Functional neuroanatomy of human rapideye-movement sleep and dreaming. Nature 383:163-166.

Marino S, Bonanno L, Giorgio A. 2016. Functional connectivity in disorders of consciousness: methodological aspects and clinical relevance. Brain Imaging Behav 10:604-608.

Martinez DE, Rudas J, Demertzi A, Charland-Verville V, Soddu A, Laureys S, Gomez F. 2020. Reconfiguration of large-scale functional connectivity in patients with disorders of consciousness. Brain Behav 10:e1476.

Martinez M, Valencia M, Vidorreta M, Luis EO, Castellanos G, Villagra F, et al. 2016. Trade-off between frequency and precision during stepping movements: kinematic and BOLD brain activation patterns. Hum Brain Mapp 37:1722-1737.

Mazaika PK, Hoeft F, Glover GH, Reiss AL. 2009. Methods and software for fMRI analysis for clinical subjects. In: Presentation at the 15th Annual Meeting of the Organization for Human Brain Mapping. Neuroimage 47:S58.

Meszlenyi RJ, Hermann P, Buza K, Gal V, Vidnyanszky Z. 2017. Resting state fMRI functional connectivity analysis using dynamic time warping. Front Neurosci 11:75.

Mitra A, Kraft A, Wright P, Acland B, Snyder AZ, Rosenthal Z, et al. 2018. Spontaneous infra-slow brain activity has unique spatiotemporal dynamics and laminar structure. Neuron 98: 297-305.

Mitra A, Raichle ME. 2016. How networks communicate: propagation patterns in spontaneous brain activity. Philos Trans R Soc Lond B Biol Sci 371. DOI: 10.1098/rstb.2015.0546

Mitra A, Raichle ME. 2018. Principles of cross-network communication in human resting state fMRI. Scand J Psychol 59:83-90. 
Mitra A, Snyder AZ, Blazey T, Raichle ME. 2015a. Lag threads organize the brain's intrinsic activity. Proc Natl Acad Sci U S A 112:E2235-2244.

Mitra A, Snyder AZ, Hacker CD, Raichle ME. 2014. Lag structure in resting-state fMRI. J Neurophysiol 111:2374-2391.

Mitra A, Snyder AZ, Tagliazucchi E, Laufs H, Raichle ME. 2015 b. Propagated infra-slow intrinsic brain activity reorganizes across wake and slow wave sleep. Elife 4. DOI: 10.7554/eLife.10781

Mohajerani MH, Chan AW, Mohsenvand M, LeDue J, Liu R, McVea DA, et al. 2013. Spontaneous cortical activity alternates between motifs defined by regional axonal projections. Nat Neurosci 16:1426-1435.

Naci L, Haugg A, MacDonald A, Anello M, Houldin E, Naqshbandi S, et al. 2018. Functional diversity of brain networks supports consciousness and verbal intelligence. Sci Rep 8:13259.

Nakayama N, Okumura A, Shinoda J, Nakashima T, Iwama T. 2006. Relationship between regional cerebral metabolism and consciousness disturbance in traumatic diffuse brain injury without large focal lesions: an FDG-PET study with statistical parametric mapping analysis. J Neurol Neurosurg Psychiatry 77:856-862.

Nikolić D. 2007. Non-parametric detection of temporal order across pairwise measurements of time delays. J Comput Neurosci 22:5-19.

Palomero-Gallagher N, Vogt BA, Schleicher A, Mayberg HS, Zilles K. 2009. Receptor architecture of human cingulate cortex: evaluation of the four-region neurobiological model. Hum Brain Mapp 30:2336-2355.

Persuh M, LaRock E, Berger J. 2018. Working memory and consciousness: the current state of play. Front Hum Neurosci 12:78.

Pistoia F, Sacco S, Stewart J, Sara M, Carolei A. 2016. Disorders of consciousness: painless or painful conditions?-evidence from neuroimaging studies. Brain Sci 6. pii: E47.

Power JD, Barnes KA, Snyder AZ, Schlaggar BL, Petersen SE. 2012. Spurious but systematic correlations in functional connectivity MRI networks arise from subject motion. Neuroimage 59:2142-2154.

Rincon AL, Shimoda S. 2016. The inverse problem in electroencephalography using the bidomain model of electrical activity. J Neurosci Methods 274:94-105.

Rudas J, Guaje J, Demertzi A, Heine L, Tshibanda L, Soddu A, et al. 2014. A method for functional network connectivity using distance correlation. Conf Proc IEEE Eng Med Biol Soc 2014:2793-2796.

Schiefer J, Niederbühl A, Pernice V, Lennartz C, Hennig J, LeVan P, Rotter S. 2018. From correlation to causation: estimating effective connectivity from zero-lag covariances of brain signals. PLoS Comput Biol 14:1-18.

Seeley W, Menon V, Schatzberg A, Keller J, Glover G, Kenna $\mathrm{H}$, et al. 2007. Dissociable intrinsic connectivity networks for salience processing and executive control. J Neurosci 27:2349-2356.

Siegel JS, Snyder AZ, Ramsey L, Shulman GL, Corbetta M. 2016. The effects of hemodynamic lag on functional connectivity and behavior after stroke. J Cereb Blood Flow Metab 36:2162-2176.

Sinitsyn DO, Legostaeva LA, Kremneva EI, Morozova SN, Poydasheva AG, Mochalova EG, et al. 2018. Degrees of functional connectome abnormality in disorders of consciousness. Human Brain Mapp 39:2929-2940.
Sitaram R, Yu T, Halsband U, Vogel D, Müller F, Lang S, et al. 2019. Spatial characteristics of spontaneous and stimulusinduced individual functional connectivity networks in severe disorders of consciousness. Brain Cogn 131:10-21.

Tak S, Noh J, Cheong C, Zeidman P, Razi A, Penny WD, Friston KJ. 2018. A validation of dynamic causal modelling for $7 \mathrm{~T}$ fMRI. J Neurosci Methods 305:36-45.

Threlkeld Z, Bodien Y, Rosenthal E, Giacino J, Nieto-Castanon $\mathrm{A}, \mathrm{Wu} \mathrm{O}$, et al. 2018. Functional networks reemerge during recovery of consciousness after acute severe traumatic brain injury. Cortex 106:299-308.

Vaishnavi SN, Vlassenko AG, Rundle MM, Snyder AZ, Mintun MA, Raichle ME. 2010. Regional aerobic glycolysis in the human brain. Proc Natl Acad Sci U S A 107: 17757-17762.

van den Heuvel MP, Pol HEH. 2010. Exploring the brain network: a review on resting-state fMRI functional connectivity. Eur Neuropsychopharmacol 20:519-534.

Vergara VM, Mayer AR, Kiehl KA, Calhoun VD. 2018. Dynamic functional network connectivity discriminates mild traumatic brain injury through machine learning. Neuroimage Clin 19:30-37.

Vodrahalli K, Chen P-H, Liang Y, Baldassano C, Chen J, Yong E, et al. 2018. Mapping between fMRI responses to movies and their natural language annotations. Neuroimage 180: 223-231.

Vogt BA. 2016. Midcingulate cortex: structure, connections, homologies, functions and diseases. J Chem Neuroanat 74:2846.

Vogt BA, Vogt L, Laureys S. 2006. Cytology and functionally correlated circuits of human posterior cingulate areas. Neuroimage 29:452-466.

Wannez S, Heine L, Thonnard M, Gosseries O, Laureys S. 2017. The repetition of behavioral assessments in diagnosis of disorders of consciousness. Ann Neurol 81:883-889.

Weng Y, Qi R, Chen F, Ke J, Xu Q, Zhong Y, et al. 2018. The temporal propagation of intrinsic brain activity associate with the occurrence of PTSD. Front Psychiatry 9:218.

You Y, Bai L, Dai R, Cheng H, Liu Z, Wei W, Tian J. 2013. Altered hub configurations within default mode network following acupuncture at ST36: a multimodal investigation combining fMRI and MEG. PLoS One 8:e64509.

Yu C, Zhou Y, Liu Y, Jiang T, Dong H, Zhang Y, Walter M. 2011. Functional segregation of the human cingulate cortex is confirmed by functional connectivity based neuroanatomical parcellation. Neuroimage 54:2571-2581.

Zimmermann M, Rössler K, Kaltenhäuser M, Grummich P, Brandner N, Buchfelder M, et al. 2019. Comparative fMRI and MEG localization of cortical sensorimotor function: bimodal mapping supports motor area reorganization in glioma patients. PLoS One 14:e0213371.

Address correspondence to: Francisco Gómez

Department of Mathematics Universidad Nacional de Colombia Street 45 \#26-85 Bogotá 111311

Colombia

E-mail: fagomezj@unal.edu.co 